Math. Model. Nat. Phenom.

Vol. 8, No. 3, 2013, pp. 33-41

DOI: $10.1051 / \mathrm{mmnp} / 20138304$

\title{
Wave-like Solutions for Nonlocal Reaction-diffusion Equations: a Toy Model
}

\author{
G. $\operatorname{Nadin}^{1 *}$, L. Rossi ${ }^{2}$, L. Ryzhik ${ }^{3}$, B. Perthame ${ }^{1}$ \\ ${ }^{1}$ Laboratoire Jacques-Louis Lions, UPMC Univ. Paris 6 and CNRS UMR 7598, F-75005, Paris \\ ${ }^{2}$ Dipartimento di Matematica, Università degli Studi di Padova \\ ${ }^{3}$ Department of Mathematics, Stanford University, Stanford CA 94305
}

\begin{abstract}
Traveling waves for the nonlocal Fisher Equation can exhibit much more complex behaviour than for the usual Fisher equation. A striking numerical observation is that a traveling wave with minimal speed can connect a dynamically unstable steady state 0 to a Turing unstable steady state 1 , see $[12]$. This is proved in $[1,6]$ in the case where the speed is far from minimal, where we expect the wave to be monotone.

Here we introduce a simplified nonlocal Fisher equation for which we can build simple analytical traveling wave solutions that exhibit various behaviours. These traveling waves, with minimal speed or not, can (i) connect monotonically 0 and 1, (ii) connect these two states non-monotonically, and (iii) connect 0 to a wavetrain around 1 . The latter exist in a regime where time dynamics converges to another object observed in $[3,8]$ : a wave that connects 0 to a pulsating wave around 1 .
\end{abstract}

Keywords and phrases: traveling wave, wavetrains, nonlocal elliptic equations

Mathematics Subject Classification: 34K13, 35B36, 35C07, 35K57

\section{Introduction}

What possible states can a traveling wave connect? The cases of two stable states, or one unstable and one stable states are standard. Here we investigate the case of a connection between a Turing unstable state (in the sense that the unstable modes are bounded and there are no highly oscillatory solutions [13]) and a dynamically unstable state using a nonlocal reaction-diffusion equation. Namely, we consider the equation:

$$
\partial_{t} u(t, x)-\partial_{x x} u(t, x)= \begin{cases}A u(t, x) & \text { for } 0 \leq u(t, x)<\theta \\ 1-u(t, x-a) & \text { for } u(t, x) \geq \theta,\end{cases}
$$

with $\theta \in(0,1), a, A>0$ and investigate various types of wave-like solutions, that is, time-global solutions which can be written as $u(t, x)=U(x-c t)$.

${ }^{*}$ Corresponding author. E-mail: nadin@ann.jussieu.fr 
This equation is a toy model aiming at understanding the dynamics of more general nonlocal reactiondiffusion equations, such as the Fisher-KPP equation with a nonlocal competition term:

$$
\partial_{t} u-\partial_{x x} u=u\left(1-\phi_{\sigma} \star u\right) \text { in } \mathbb{R} \times \mathbb{R},
$$

where $\phi_{\sigma}(z)=\frac{1}{\sigma} \phi(z / \sigma), \phi \in L^{1}(\mathbb{R})$ is a smooth nonnegative kernel such that $\phi(0)>0$ and $x^{2} \phi(x) \in$ $L^{1}(\mathbb{R}), \sigma>0$ is a parameter measuring the lenght of the nonlocality and $\star$ is the convolution product. This equation arises in various areas such as ecology [7] or adaptative dynamics [8] (see also [4, 10]). It admits a positive solution of the form $u(t, x)=U(x-c t)$, with $U(+\infty)=0$ and $\liminf _{x \rightarrow-\infty} U>0$, for all $c \geq 2$ [3]. Moreover, if the Fourier transform of $\phi$ is positive, for example if $\phi$ is a Gaussian kernel, then $U(-\infty)=1[3]$. However, such a convergence is not known in general.

Numerical simulations of the evolution equation (1.2) show that nontrivial steady states may exist when $\sigma$ is large enough $[3,8]$. That is, there might exist periodic positive solutions $p(x)$ of

$$
-p^{\prime \prime}=p\left(1-\phi_{\sigma} \star p\right)
$$

over $\mathbb{R}$. These numerical simulations also suggest that these steady states could be connected to 0 through pulsating traveling waves. Due to the nonlocal term $\phi_{\sigma} \star u$, the steady state 1 could be linearly unstable with respect to some periodic perturbations, creating a bifurcation branch of stable periodic steady states. By analogy with reaction-diffusion systems, where similar phenomena occur, we call it a Turing mecanism and we then say that the steady state 1 is Turing unstable. Note that such a mecanism cannot arise if one considers the nonlinearity $\left(\phi_{\sigma} \star u\right)(1-u)$ instead of $u\left(1-\phi_{\sigma} \star u\right)$ since in this case a comparison principle holds, forbidding the existence of positive nontrivial steady states. Hence, these two nonlinearities are very different, from an analytical as well as a qualitative point of view.

The existence of traveling waves connecting 0 to 1 , that is, solutions $(c, U) \in \mathbb{R} \times \mathcal{C}^{2}(\mathbb{R})$ of

$$
\begin{cases}-U^{\prime \prime}-c U^{\prime}=U\left(1-\phi_{\sigma} \star U\right) & \text { in } \mathbb{R} \\ U(-\infty)=1, \quad U(+\infty)=0, & U>0\end{cases}
$$

remains unclear when the steady state 1 is Turing unstable. An alternative numerical approach was developed in [12] in order to approximate the equation satisfied by the traveling waves (1.3) instead of the evolution equation (1.1), giving different results. Namely, these numerical simulations suggest that positive traveling waves necessarily connect 0 to 1 . This is quite surprising, since for the classical (local) Fisher-KPP equation or more generally for reaction-diffusion equations admitting a comparison principle, unstable steady states can only be connected to stable ones.

The existence of monotone traveling waves for (1.3) was recently investigated by Fang and Zhao [6]. They proved that for all $c \geq 2$, there exists an explicit threshold parameter $\sigma_{c} \in(0, \infty]$, such that there exists a decreasing traveling wave solution of equation (1.3) if and only if $\sigma \in\left(0, \sigma_{c}\right)$. The convergence of this wave to 1 at $-\infty$ immediatly follows from the monotonicity. This work was inspired by earlier papers on reaction-diffusion equations with delayed competition effect [9,11], which exhibit similar phenomena. As a corollary of this result, not mentioned explicitly in [6], one could derive the existence of traveling waves connecting the Turing unstable steady state 1 to the dynamically unstable one 0 . Indeed, if the Fourier transform $\hat{\phi}$ is not nonnegative, then when $\sigma$ is large enough, the steady state 1 is linearly unstable with respect to perturbations of some given period range (see $[3,8]$ ). On the other hand, it easily follows from the explicit expression of $\sigma_{c}$ that $\lim _{c \rightarrow+\infty} \sigma_{c}=+\infty$. Hence, there always exists some sufficiently large speed $c$ such that $\sigma<\sigma_{c}$, and thus there exists a traveling wave of speed $c$ connecting 0 to 1 monotonically. The fact that traveling waves with large speeds necessarily converge to 1 at $-\infty$ was also proved through direct arguments by Alfaro and Coville [1]. It is not clear to us whether the threshold on the speed provided in [1] necessarily implies that the wave is monotone or not.

If the case of monotonic traveling wave solutions of (1.2) is now fully understood, the existence of non-monotonic traveling waves and their convergence to 1 is still unclear. Numerical simulations [12] and the case of large speeds [1] suggest that the convergence to 1 always hold, whatever the Turing 
stability of this state is. On the other hand, in the present paper, investigating the toy model (1.1) we find completely different results: many different types of wave-like solutions, that is, solutions that are stationary in a moving frame with a given speed $c$, can be constructed. This equation not only admits monotonic traveling waves but also non-monotonic ones converging to 1 , wave-trains oscillating around 1 and traveling waves connecting 0 to these wavetrains. Hence, our present results suggest that, similarly, equation (1.2) admits many types of wave-like solutions. In order to avoid any possible confusion, we underline that we do not consider pulsating traveling waves here, that is, solutions that are time-periodic in a moving frame.

\section{Statement of the results}

\subsection{Monotone traveling waves connecting 0 to 1 .}

First, the sign of the spatial nonlocality $a>0$ ensures that monotone traveling waves $u(x, t)=U(x-c t)$ always exist. The profile $U$ satisfies $U>0$ in $\mathbb{R}, U(-\infty)=1, U(+\infty)=0$ and

$$
-U^{\prime \prime}(z)-c U^{\prime}(z)=\left\{\begin{array}{ll}
A U(z) & \text { for } 0 \leq U(z)<\theta \\
1-U(z-a) & \text { for } \theta \leq U(z)
\end{array} \quad \text { over } \mathbb{R}\right.
$$

Proposition 2.1. Assume that $A \geq(1-\theta) / \theta$. For all $c \geq 2 \sqrt{A}$, there exists $U \in W^{2, \infty}(\mathbb{R})$ satisfying (2.1) such that $U$ is decreasing, $U(+\infty)=0$ and $U(-\infty)=1$.

Note that the condition $A \geq(1-\theta) / \theta$ is the equivalent of a KPP condition for the discontinuous equation (1.1): if $a=0$, it means that the nonlinearity is below its tangent $A$ at $u=0$. If this assumption is relaxed, the reader could check that our method still applies, giving the existence of traveling waves with speeds above a larger and less explicit threshold.

This result is not surprising. If the nonlinearity in (1.1) were continuous, then it would be an immediate consequence of Fang-Zhao's condition for the existence of monotonic traveling waves [6] since the linearization of the equation near $u=1$ always admits real exponential solutions. Our proof relies on an explicit construction of the traveling wave.

\subsection{Oscillating traveling waves connecting 0 to 1 .}

The next result states that oscillating traveling waves connecting 0 to 1 exist when the delay $a$ is large enough.

Proposition 2.2. Assume that $a>0$ and $c \geq 2 \sqrt{A}$ satisfy

$$
\begin{aligned}
& c \leq \frac{2 a}{3 \pi} e^{-\frac{3}{2} \pi}-\frac{3 \pi}{a}, \\
& \frac{2 a}{3 \pi} \leq(c+2 / c) e^{a / c} .
\end{aligned}
$$

Then there exists a positive bounded solution $U \in W^{2, \infty}(\mathbb{R})$ of (2.1) such that $U(-\infty)=1, U(+\infty)=0$ and $U$ is not monotone.

One can easily check that these two inequalities both hold for $a$ large enough. This result is more surprising since one would have expected oscillating traveling waves to appear only when the condition ensuring the monotonicty in [6] could not be satisfied anymore (see the numerical simulations in $[3,8,12]$ ). On the contrary, Propositions 2.1 and 2.2 show that monotonic and oscillating traveling waves can coexist. 

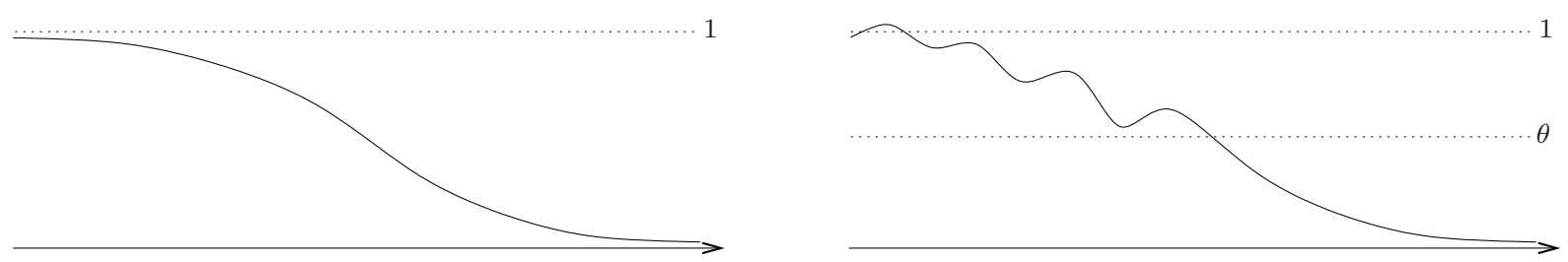

Figure 1. Monotone (left) and and oscillating (right) traveling waves connecting 0 to 1.

\subsection{Wavetrains periodic around 1 .}

As for reaction-diffusion systems (see for example [5]), we now construct a new type of solutions.

Definition 2.3. We say that a positive solution $u$ of (1.1) is a wavetrain of speed $c$ if it can be written as $u(t, x)=W(x-c t)$, where $W \in W^{2, \infty}(\mathbb{R})$ is positive and periodic, that is, there exists $L>0$ such that $W(z+L)=W(z)$ for all $z \in \mathbb{R}$. We call $W$ the profile of the wavetrain $u$. Lastly, we say that a wave train $u$ is nontrivial if $u \not \equiv 1$.

Such solutions have been observed numerically in the framework of nonlocal reaction-diffusion equations with asymmetric kernels in $[2,8]$.

The profile $W$ of a wavetrain of speed $c$ is a weak solution of

$$
-W^{\prime \prime}(z)-c W^{\prime}(z)=\left\{\begin{array}{cl}
A W(z) & \text { if } 0<W(z)<\theta \\
1-W(z-a) & \text { if } W(z) \geq \theta
\end{array}\right.
$$

Proposition 2.4. Assume that $(2 k+1) \pi \leq a<(2 k+3) \pi$, with $k \in \mathbb{N}$. Then equation (1.1) admits $k+1$ nontrivial wavetrains of the form

$$
u_{i}(x, t)=1+V_{i}\left(x-c_{i} t\right), \quad i=1, \ldots, k+1,
$$

with $c_{1}>\cdots>c_{k+1}>0$. Moreover, any wavetrain $u$ of (1.1) satisfies

$$
\theta \leq u \leq 2-\theta, \quad u(x, t)=1+C V_{i}\left(x-c_{i} t+\varphi\right),
$$

for some $i \in\{1, \ldots, k+1\}$ and $C, \varphi \in \mathbb{R}$.

Note that any convex combination of such wavetrains $u_{1}, \ldots, u_{k+1}$ is still a time-global solution of (1.1) connecting 0 to 1 . However, it is not a wavetrain anymore nor a wave-like solution since the $u_{i}$ have different speeds $c_{1}>\ldots>c_{k+1}$.

\subsection{Connections between 0 and wavetrains}

Lastly, we use the wavetrains to construct solutions of the form $u(t, x)=U(x-c t)$, with $U>0$ converging to 0 at $+\infty$ and not converging to 1 at $-\infty$, but to a wavetrain.

Proposition 2.5. For all $a \geq a_{*}:=\frac{3}{2} \pi \sqrt{2 A+\sqrt{4 A^{2}+1}}$, there exists a solution $u$ of (1.1) which can be written as $u(t, x)=U(x-c t)$, with $c>0$ and $U \in W^{2, \infty}(\mathbb{R})$ a positive function such that $\lim _{z \rightarrow+\infty} U(z)=0$ and $\lim _{z \rightarrow-\infty}(U(z)-W(z))=0$, where $W$ is the profile of a nontrivial wavetrain of speed $c$.

Such a solution will not be unique in general. Indeed, we know from Proposition 2.4 that there may exist several wavetrains and, adapting the proof below, one might construct different traveling waves connecting 0 to each of these wavetrains, under some implicit conditions on $a$. We leave such a classification to forthcoming work. 

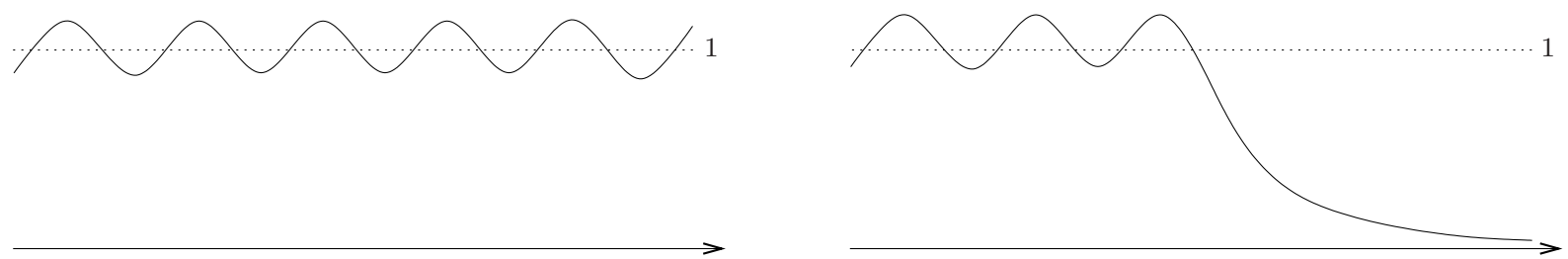

Figure 2. Wavetrain (left) and its connection to 0 (right).

\section{Proofs of the results}

\subsection{Construction of the monotone traveling waves}

Proof of Proposition 2.1. Let $\lambda>0$ be the unique positive solution of $f_{c}(\lambda):=\lambda^{2}+\lambda c-e^{-a \lambda}=0$, which is unique by monotonicity. The positivity of $a$ ensures that such a solution always exists.

Next, as $c \geq 2 \sqrt{A}$, the polynomial equation $\eta^{2}-\eta c+A=0$ admits two solutions $0<\eta_{-} \leq \eta_{+}$(if $c=2 \sqrt{A}$, then $\eta_{+}=\eta_{-}=\sqrt{A}$ ). For all $z \in \mathbb{R}$ and for $\alpha>0$ to be fixed later, let

$$
V(z):=\left\{\begin{array}{cc}
(\theta+\alpha) e^{-\eta_{-} z}-\alpha e^{-\eta_{+} z} & \text { if } c>2 \sqrt{A} \\
\theta(1+\alpha z) e^{-\eta_{-} z} & \text { if } c=2 \sqrt{A}
\end{array}\right.
$$

It is easy to check that, if $\alpha>0$, with the additional assumption $\alpha \leq \eta_{-}$when $c=2 \sqrt{A}$, then

$$
-V^{\prime \prime}(z)-c V^{\prime}(z)=A V(z)
$$

for all $z>0$. We compute

$$
V^{\prime}(0)=\left\{\begin{array}{cl}
\alpha\left(\eta_{+}-\eta_{-}\right)-\eta_{-} \theta & \text { if } c>2 \sqrt{A} \\
\theta\left(\alpha-\eta_{-}\right) & \text {if } c=2 \sqrt{A}
\end{array}\right.
$$

Let $\alpha \in \mathbb{R}$ be such that $V^{\prime}(0)=-\lambda(1-\theta)$. The condition $\alpha \leq \eta_{-}$is clearly satisfied since $V^{\prime}(0) \leq 0$. We now need to check that $\alpha$ is positive. A straightforward computation gives that, in both cases $c=2 \sqrt{A}$ and $c>2 \sqrt{A}, \alpha>0$ if and only if

$$
\theta \eta_{-}>\lambda(1-\theta)
$$

As $A \geq(1-\theta) / \theta$, a sufficient condition for the positivity of $\alpha$ is $\lambda<\eta_{-} / A$. On the other hand,

$$
\begin{aligned}
f_{c}\left(\eta_{-} / A\right) & =\frac{\eta_{-}^{2}}{A^{2}}+\frac{c \eta_{-}}{A}-e^{-\frac{a \eta_{-}}{A}} \\
& \geq \frac{\eta_{-}^{2}}{A^{2}}+\frac{c \eta_{-}}{A}-1 \\
& =\frac{\eta_{-}^{2}}{A^{2}}+\frac{\eta_{-}^{2}}{A}-A \\
A & =\frac{\eta_{-}^{2}}{A^{2}}(1+A)>0 .
\end{aligned}
$$

As $\lambda$ is the unique positive root of the increasing function $f_{c}$, one gets $\eta_{-} / A>\lambda$, from which we eventually derive $\alpha>0$.

Define for all $z \in \mathbb{R}$ :

$$
U(z):=\left\{\begin{array}{cc}
1-(1-\theta) e^{\lambda z} & \text { if } z \leq 0 \\
V(z) & \text { if } z>0
\end{array}\right.
$$

This function is clearly decreasing and converges to 1 at $-\infty$ and 0 at $+\infty$. It is easy to check that $U$ satisfies $(2.1)$ on $(-\infty, 0)$ and on $(0, \infty)$. Moreover, we have constructed $V$ in such a way that $V(0)=\theta$ and $V^{\prime}(0)=-\lambda(1-\theta)$, meaning that $U \in \mathcal{C}^{1}(\mathbb{R})$. Hence, $U$ is a weak solution of (2.1) over $\mathbb{R}$. 


\subsection{Construction of the oscillating traveling waves}

Lemma 3.1. For all $\lambda>0$, if

$$
e^{a \lambda}(c+2 \lambda) \leq \frac{2 a}{3 \pi},
$$

then there exists a unique $\mu(\lambda) \in\left[\frac{3 \pi}{2 a}, \frac{2 \pi}{a}\right)$ such that

$$
-\frac{\sin (a \mu(\lambda))}{\mu(\lambda)}=e^{a \lambda}(c+2 \lambda)
$$

Moreover, the function $\mu$ is continuous (where it is defined).

Proof. Clearly, $\mu \mapsto-\sin (a \mu) / \mu$ is decreasing over $\left[\frac{3 \pi}{2 a}, \frac{2 \pi}{a}\right]$. As

$$
-\frac{\sin \left(a \times \frac{3 \pi}{2 a}\right)}{\frac{3 \pi}{2 a}}=\frac{2 a}{3 \pi} \geq e^{a \lambda}(c+2 \lambda) \quad \text { and } \quad-\frac{\sin \left(a \times \frac{\pi}{a}\right)}{\frac{\pi}{a}}=0<e^{a \lambda}(c+2 \lambda),
$$

the intermediate value theorem gives the existence. The monotonicity gives the uniqueness, from which the continuity easily follows.

Proof of Proposition 2.2. As $c<\frac{2 a}{3 \pi}$ by (2.2), we can define $\Lambda$ as the unique positive solution of

$$
e^{a \Lambda}(c+2 \Lambda)=\frac{2 a}{3 \pi} .
$$

Then for all $\lambda \in[0, \Lambda],(3.4)$ is satisfied and thus $\mu(\lambda)$ is well-defined. Moreover, $\mu(\Lambda)=3 \pi / 2 a$ by uniqueness. Let

$$
f(\lambda):=\lambda^{2}+c \lambda-\mu(\lambda)^{2}-e^{-a \lambda} \cos (a \mu(\lambda)) .
$$

This function is continuous and one has

$$
f(0)=-\mu(0)^{2}-\cos (a \mu(0))<0
$$

since $\mu(0) \in\left[\frac{3 \pi}{2 a}, \frac{2 \pi}{a}\right]$. Moreover,

$$
\begin{aligned}
f(\Lambda) & =\Lambda^{2}+c \Lambda-\mu(\Lambda)^{2}-e^{-a \Lambda} \cos (a \mu(\Lambda)) \\
& =\Lambda^{2}+c \Lambda-\left(\frac{3 \pi}{2 a}\right)^{2} \\
& >\Lambda^{2}-\left(\frac{3 \pi}{2 a}\right)^{2} .
\end{aligned}
$$

Hence, $f(\Lambda)>0$ if $\Lambda \geq 3 \pi / 2 a$. On the other hand, the increasing function $g: \lambda \mapsto e^{a \lambda}(c+2 \lambda)-2 a / 3 \pi$ vanishes at $\Lambda$ and $(2.2)$ gives

$$
g(3 \pi / 2 a)=e^{3 \pi / 2}(c+3 \pi / a)-2 a / 3 \pi \leq 0 .
$$

It follows that $\Lambda \geq 3 \pi / 2 a$ by monotonicity and thus $f(\Lambda)>0$.

The intermediate value theorem implies that there exists $\lambda \in(0, \Lambda)$ such that $f(\lambda)=0$. Defining $\mu:=\mu(\lambda)$, we have thus found a couple $(\lambda, \mu) \in(0, \infty)^{2}$ satisfying

$$
\left\{\begin{aligned}
c \lambda+\lambda^{2}-\mu^{2} & =e^{-a \lambda} \cos (a \mu) \\
\mu(c+2 \lambda) & =-e^{-a \lambda} \sin (a \mu) .
\end{aligned}\right.
$$


Next, as in the proof of Proposition 2.1, we search for $U$ in the form

$$
U(z)= \begin{cases}1-(1-\theta) e^{\lambda z} \cos (\mu z) & \text { for } z \leq 0 \\ V(z) & \text { for } z \geq 0 .\end{cases}
$$

Our choice of $(\lambda, \mu)$ and the definition (3.1) of $V$ yield that $U$ satisfies (2.1) over $\mathbb{R} \backslash\{0\}$. As in the proof of Proposition 2.1, it only remains to check that $\eta_{-}>A \lambda$ in order to be able to choose $\alpha>0$ so that $U$ is of class $W^{2, \infty}$, where $\eta_{-}$is the smallest solution of $\eta^{2}-c \eta+A=0$. It would thus follow that $U$ is a weak solution of $(2.1)$ over $\mathbb{R}$, which would conclude the proof since $U(-\infty)=1$ and $U(+\infty)=0$ are clearly satisfied.

First, as $0=\eta_{-}^{2}-c \eta_{-}+A>A-c \eta_{-}$, one has $\eta_{-}>A / c$. Whence $\eta_{-}>A \lambda$ if $\lambda<1 / c$. Since $\lambda<\Lambda$, it is then sufficient to have $\Lambda \leq 1 / c$. The latter holds true because, by (2.3),

$$
g(1 / c)=(c+2 / c) e^{a / c}-\frac{2 a}{3 \pi} \geq 0=g(\Lambda) .
$$

Thus, we can choose $\alpha>0$ so that $U$ is of class $\mathcal{C}^{1}$ and conclude the proof.

\subsection{Construction of the wavetrains}

Lemma 3.2. Assume that $u$ is a wavetrain of equation (1.1). Then $u(t, x) \geq \theta$ for all $(t, x) \in \mathbb{R} \times \mathbb{R}$.

Proof. Writing $u(t, x)=W(x-c t)$, as $W$ is periodic, we know that it admits a minimum at some point $z_{0} \in \mathbb{R}$. If $W\left(z_{0}\right)<\theta$, then (2.4) yields that $W$ is $\mathcal{C}^{2}$ in the neighborhood of $z_{0}$. As it is positive, one has: $0 \geq-W^{\prime \prime}\left(z_{0}\right)-c W^{\prime}\left(z_{0}\right)=A W\left(z_{0}\right)>0$, a contradiction.

Proof of Proposition 2.4. 1. We look for a solution $W$ of $(2.1)$ of the form $W(z)=\operatorname{Re}(V(z))$, with

$$
V(z)=1-C e^{i \xi z}, \quad \xi \in \mathbb{R}, \quad C \in(0,1-\theta] .
$$

Since $W \geq \theta$, equation $(2.1)$ reduces to

$$
-W^{\prime \prime}(z)-c W^{\prime}(z)=1-W(z-a) \text { in } \mathbb{R} .
$$

The complex-valued function $V$ is a solution of the above equation if and only if

$$
-\xi^{2}+c \xi i=e^{-i a \xi}
$$

Computing the modulus of both sides we infer that

$$
0<\xi \leq 1, \quad c^{2} \xi^{2}+\xi^{4}=1,
$$

from which we get the expression of $c$ as a function of $\xi: c(\xi)=\sqrt{\xi^{-2}-\xi^{2}}$. We thus look for solutions $\xi \in(0,1]$ of the equation $\Gamma(\xi)=\Gamma_{a}(\xi)$, where

$$
\Gamma(\xi):=-\xi^{2}+c(\xi) \xi i, \quad \Gamma_{a}(\xi):=e^{-i a \xi}
$$

are sectors of the unit circle in the complex plane, the first one parametrized counter-clockwise from the angle $\pi / 2$ to $\pi$, the second one clockwise from the angle 0 to $-a$. It is then clear that for $(2 k+1) \pi \leq$ $a<(2 k+3) \pi$, the number of solutions is exactly $k+1$ (see Figure 3). Notice that the associated $c$ 's are distinct because $\xi \mapsto c(\xi)$ is decreasing.

2. Next, if $u$ is a wavetrain of speed $c$, then Lemma 3.2 yields that $u \geq \theta$. Hence, $u$ satisfies

$$
\partial_{t} u-\partial_{x x} u=1-u(t, x-a) .
$$




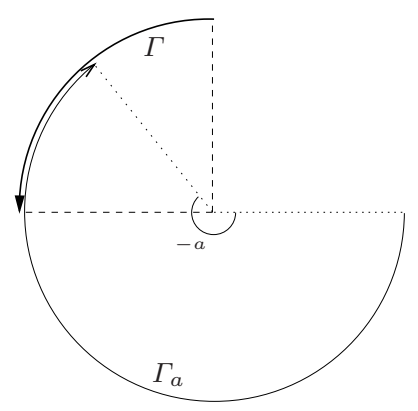

Figure 3 . The curves $\Gamma$ and $\Gamma_{a}$.

Writing $u(t, x)=1+V(x-c t)$, with $V$ periodic, we get that $(c, V)$ is a solution of

$$
V^{\prime \prime}(z)+c V^{\prime}(z)=V(z-a) \quad \text { in } \mathbb{R} .
$$

Let $L=2 \pi / \xi$ the period of $V$. Then developing $V$ in Fourier series, a straightforward computation yields that $V(z)=C \cos (\xi z+\varphi)$, with $C \in \mathbb{R}, \varphi \in \mathbb{R}$ and $\xi$ satisfying (3.7). As $c>0$, it follows from Step 1 that, up to translation by $\varphi$ and multiplication by $C, V$ is one of the wavetrains constructed in Step 1.

\subsection{Construction of the connections between 0 and the wavetrains}

Proof of Proposition 2.5. Let $a \geq \pi$. Our aim is to connect the fastest wavetrain $u_{1}$ given by Proposition 2.4 with a function $V$ of the form (3.1). As $V$ is defined only for $c \geq 2 \sqrt{A}$, we need the speed $c_{1}$ of $u_{1}$ to satisfy $c_{1} \geq 2 \sqrt{A}$. With the same notation as in the proof of Proposition 2.4, we have that the decreasing monotonicity of $\xi \mapsto c(\xi)$ implies that $c_{1}=c\left(\xi_{1}\right)$, where $\xi_{1}$ is the smallest solution of $\Gamma(\xi)=\Gamma_{a}(\xi)$ in $(0,1]$. Since $\Gamma_{a}(3 \pi / 2 a)=i$, it is clear that $\xi_{1}<3 \pi / 2 a$. Whence,

$$
c_{1}=c\left(\xi_{1}\right)>c(3 \pi / 2 a)=\sqrt{\left(\frac{2 a}{3 \pi}\right)^{2}-\left(\frac{3 \pi}{2 a}\right)^{2}} .
$$

A straightforward computation shows that the latter term is larger than or equal to $2 \sqrt{A}$ if and only if

$$
a \geq a_{*}=\frac{3}{2} \pi \sqrt{2 A+\sqrt{4 A^{2}+1}} .
$$

As a consequence, for $a \geq a_{*}$, we can define $V$ as in (3.1), with $c=c_{1}$ and $\alpha>0$ to be chosen later.

The profile of $u_{1}$ is $W(z)=1-(1-\theta) \cos \left(\xi_{1} z\right)$. Let

$$
U(z):= \begin{cases}V(z) & \text { if } z \geq 0 \\ W(z) & \text { if } z<0\end{cases}
$$

Choosing $\alpha>0$ in such a way that $V^{\prime}(0)=0$, we have that $U \in \mathcal{C}^{1}(\mathbb{R})$. Note that in the case $c=2 \sqrt{A}$, we have $\theta\left(\alpha-\eta_{-}\right)=V^{\prime}(0)=0$ and thus the condition $\alpha \leq \eta_{-}$is still satisfied. Hence, $u(t, x):=U(x-c t)$ satisfies the required properties.

Acknowledgements. Luca Rossi was supported by GNAMPA - INdAM (Italy) and the Fondazione CaRiPaRo Project "Nonlinear Partial Differential Equations: models, analysis, and control-theoretic problems". The research leading to these results has received funding from the European Research Council under the European Union's Seventh Framework Programme (FP/2007-2013) / ERC Grant Agreement n.321186 - ReaDi -Reaction-Diffusion Equations, Propagation and Modelling. 


\section{References}

[1] M. Alfaro, J. Coville. Rapid traveling waves in the nonlocal Fisher equation connect two unstable states. Appl. Math. Lett., 25:2095-2099, 2012.

[2] N. Apreutesei, N. Bessonov, V. Volpert, V. Vougalter. Spatial structures and generalized travelling waves for an integro-differential equation. Disc. Cont. Dyn. Syst. B, 13(3):537-557, 2010.

[3] H. Berestycki, G. Nadin, B. Perthame, L. Ryzhik. The non-local Fisher-KPP equation: traveling waves and steady states. Nonlinearity, 22(12):2813-2844, 2009.

[4] N. Britton. Spatial structures and periodic traveling waves in an integro-differential reaction-diffusion population model. SIAM J. Appl. Math., 50(6):1663-1688, 1990.

[5] A. Doelman, B. Sandstede, A. Scheel, G. Schneider. The dynamics of modulated wave trains. Mem. Amer. Math. Soc., 199(934), 2009.

[6] J. Fang, X-Q. Zhao. Monotone wavefronts of the nonlocal Fisher-KPP equation. Nonlinearity, 24(11):3043-3054, 2011.

[7] J-É Furter, M. Grinfeld. Local vs. nonlocal interactions in population dynamics. J. Math. Biol., 27(1):65-80, 1989.

[8] S. Genieys, V. Volpert, P. Auger. Pattern and waves for a model in population dynamics with nonlocal consumption of resources. Math. Modelling Nat. Phenom., 1:65-82, 2006.

[9] A. Gomez, S. Trofimchuk. Monotone traveling wavefronts of the KPP-Fisher delayed equation. J. Diff. Eq., 250(4):17671787, 2011.

[10] S. Gourley. Traveling front solutions of a nonlocal Fisher equation. J. Math. Biol., 41(3):272-284, 2000.

[11] M.K. Kwong, C. Ou. Existence and nonexistence of monotone traveling waves for the delayed Fisher equation. J. Diff. Eq., 249(3):728-745, 2010.

[12] G. Nadin, B. Perthame, M. Tang. Can a traveling wave connect two unstable states? The case of the nonlocal Fisher equation. C. R. Math. Acad. Sci. Paris, 349(9-10):553-557, 2011.

[13] A. Turing. The chemical basis of morphogenesis. Phil. Trans. Royal Soc. London. Serie B, Biol. Sc., 237(641):37-72, 1952. 